\title{
The variational iteration method for solving $n$-th order fuzzy differential equations
}

Research Article

\author{
Hossein Jafari ${ }^{1 *}$, Mohammad Saeidy ${ }^{1}$, Dumitru Baleanu ${ }^{2}$ \\ 1 Department of Mathematics and Computer Science,University of Mazandaran, \\ P. O. Box 47416-1467, Babolsar, Iran \\ 2 Department of Mathematics and Computer Science \\ Çankaya University, 06530 Balgat, Ankara, Turkey ${ }^{\dagger}$
}

Received 25 July 2011 ; accepted 14 September 2011

Abstract: The variational iteration method (VIM) proposed by Ji-Huan He is a new analytical method for solving linear and nonlinear equations. In this paper, the variational iteration method has been applied in solving $n$ thorder fuzzy linear differential equations with fuzzy initial conditions. This method is illustrated by solving several examples.

PACS (2008): 02.60.Lj; 07.05.Mh; 02.60.Cb; 02.90.+p

Keywords: $\quad$ variational iteration method $\bullet$ fuzzy number $\bullet n$ th-order fuzzy linear differential equation

(C) Versita Sp. z o.o.

\section{Introduction}

The topic of fuzzy differential equations (FDEs) has been rapidly growing in recent years. The concept of the fuzzy derivative was first introduced by Chang and Zadeh[32]; it was followed up by Dubois and Prade [16], who used the extension principle in their approach. Other methods have been discussed by Puri and Ralescu [17] and

*E-mail: jafari@umz.ac.ir

${ }^{+}$On leave of absence from Institute of Space Sciences,

P.O.BOX, MAG-23, R 76900, Magurele-Bucharest, Romania
Goetschel and Voxman [29]. Kandel and Byatt [1, 2] applied the concept of fuzzy differential equation (FDE) to the analysis of fuzzy dynamical problems. The FDE and the initial value problem (Cauchy problem) were rigorously treated by Kaleva [23, 24, 27], Seikkala [31], He and $\mathrm{Yi}$ [26], Kloeden [28] and Menda [36], and by other researchers (see $[15,19-22,25,35])$. The numerical methods for solving fuzzy differential equations were introduced in $[30,34]$. Buckley and Feuring [18] introduced two analytical methods for solving $n$-th order linear differential equations with fuzzy initial conditions. Their first method of solution was to fuzzify the crisp solution and then check 
to see if it satisfies the differential equation with fuzzy initial conditions; and the second method was the reverse of the first method, in that they first solved the fuzzy initial value problem and the checked to see if it defined a fuzzy function.

Analytical methods commonly used to solve linear equations are very restricted. On the other hand, numerical techniques involving discretization of the variables give rise to rounding errors. A recently introduced variational iteration method (VIM) by $\mathrm{He}$ [7-10], which gives rapidly convergent successive approximations of the exact solution if such a solution exists, has proven successful in deriving analytical solutions of linear and nonlinear differential equations. This method is preferable over numerical methods as it is free from rounding errors and requires neither large computer power nor a lot of memory. He $[8,9,11]$ has applied this method for obtaining analytical solutions of autonomous ordinary differential equation, linear partial differential equations with variable coefficients and integro-differential equations. The VIM was successfully applied to the Burgers and coupled Burgers equations [3]; to the Schrödinger- $K d V$, generalized $K d V$, and shallow water equations [4]; and to the linear Helmholtz partial differential equation [5]. The linear and nonlinear wave equations, $K d V, K(2,2)$, Burgers, and cubic Boussinesq equations have been solved by Wazwaz $[13,14]$ using the variational iteration method.

We set two goals for this paper. First, we aim to employ the VIM to effectively handle $n$-th order fuzzy differential equations. Second, the study will be supported by numerical examples to confirm our belief in the effectiveness of the method.

\section{Preliminaries}

In this section the most basic notations used in fuzzy calculus are introduced. We start with defining a fuzzy number.

\section{Definition 1.}

A fuzzy number is a fuzzy set $u: R^{1} \longrightarrow I=[0,1]$ which satisfies

(i) $u$ is upper semicontinuous. (ii) $u(x)=0$ outside some interval $[c, d]$.

(iii) There are real numbers $a, b: c \leq a \leq b \leq d$ for which

1. $u(x)$ is monotonic increasing on $[c, a]$,

2. $u(x)$ is monotonic decreasing on $[b, d]$,

3. $u(x)=1, a \leq x \leq b$.

The set of all fuzzy numbers (as given by Definition 1) is denoted by $E^{1}$. An alternative definition or parametric form of a fuzzy number which yields the same $E^{1}$ is given by Kaleva [27].

\section{Definition 2.}

A fuzzy number $u$ is a pair $(\underline{u}, \bar{u})$ of functions $\underline{u}(r), \bar{u}(r) ; 0 \leq$ $r \leq 1$ which satisfying the following requirements:

i. $\underline{u}(r)$ is a bounded monotonic increasing left continuous function,

ii. $\bar{u}(r)$ is a bounded monotonic decreasing left continuous function,

iii. $\underline{u}(r) \leq \bar{u}(r), 0 \leq r \leq 1$.

For arbitrary $u=(\underline{u}, \bar{u}), v=(\underline{v}, \bar{v})$ and $k>0$ we define addition $(u+v)$ and multiplication by $k$ as

$$
\begin{aligned}
& (\underline{u+v})(r)=\underline{u}(r)+\underline{v}(r), \\
& (\overline{u+v)}(r)=\bar{u}(r)+\bar{v}(r),
\end{aligned}
$$

$$
(\underline{k u})(r)=k \underline{u}(r), \quad(\overline{k u})(r)=k \bar{u}(r) .
$$

The collection of all the fuzzy numbers with addition and multiplication as defined by Eqs. (1) and (2) is denoted by $E^{1}$ and is a convex cone. It can be shown that Eqs. (1) and (2) are equivalent to the addition and multiplication as defined by using the $\alpha$-cut approach [16] and the extension principles [6]. We will next define the fuzzy function notation and a metric $D$ in $E^{1}[16]$. 


\section{Definition 3.}

[33] For arbitrary fuzzy numbers $u=(\underline{u}, \bar{u})$ and $v=(\underline{v}, \bar{v})$ the quantity

$$
D(u, v)=\left[\int_{0}^{1}(\underline{u}(r)-\underline{v}(r))^{2} d r+\int_{0}^{1}(\bar{u}(r)-\bar{v}(r))^{2} d r\right]^{\frac{1}{2}}
$$

is the distance between $u$ and $v$.

\section{He's variational iteration method}

To illustrate the basic concept of He's variational iteration method consider the following general nonlinear equation:

$$
\mathcal{L} u(t)+\mathcal{N} u(t)=g(t)
$$

where $\mathcal{L}, \mathcal{N}$ are linear and nonlinear operators, respectively, and $g(t)$ is the source inhomogeneous term. He has modified the general Lagrange multiplier method to an iteration method known as correction functional. The basic character of the method is to construct a correction functional for the above equation, which reads as

$$
u_{n+1}(t)=u_{n}(t)+\int_{0}^{t} \lambda\left(\mathcal{L} u_{n}+\mathcal{N} \widetilde{u}_{n}-g(s)\right) d s,
$$

where $\lambda$ is a general Lagrange multiplier, which can be identified optimally via the variational theory. The subscript $n$ denotes the $n$th approximation, and $\widetilde{u}_{n}$ is a restricted variation i.e. $\delta \widetilde{u}_{n}=0$. It is to be noted that the Lagrange multiplier $\lambda$ can be constant or a function.

First, it is required to determine the Lagrange multiplier $\lambda$ that can be identified optimally via integration by parts and by using a restricted variation. It was found in $[37,38]$ that a general formula for $\lambda$ for the $n$-th order differential equation

$$
y^{(n)}+f\left(y(t), y^{\prime}(t), y^{\prime \prime}(t), \ldots, y^{(n)}(t)\right)=0
$$

can be proved to be of the form

$$
\lambda=(-1)^{n} \frac{(s-t)^{(n-1)}}{(n-1) !}
$$

Having determined the Lagrange multiplier, the successive approximations $u_{n+1}$ will be calculated by using any initial function $u_{0}$. Consequently, the solution is obtained by taking the limit:

$$
u=\lim _{n \rightarrow \infty} u_{n}
$$

In other words, the corrections functional (5) will give a sequence of approximations and the exact solution is obtained at the limit of the successive approximations. In fact, the solution of problem (4) is considered as a fixed point of the following functional under a suitable choice of the initial term $u_{0}(t)$ :

$$
u_{n+1}=u_{n}+\int_{0}^{t} \lambda\left(\mathcal{L} u_{n}+\mathcal{N} u_{n}-g(s)\right) d s
$$

As a well-known powerful tool, we have:

\section{Theorem 1.}

(Banach's Fixed Point Theorem). Assume that $X$ is a Banach space and

$A: X \rightarrow X$ is a nonlinear mapping, and suppose that $\|A[u]-A[v]\| \leq k\|u-v\|, \quad u, v \in X$

for some constants $k<1$. Then $A$ has a unique fixed point. Furthermore, the sequence $u_{n+1}=A\left[u_{n}\right]$, with an arbitrary choice of $u_{0} \in X$, converges to the fixed point of A.

According to Theorem (1), for the nonlinear mapping

$$
A[u(t)]=u(t)+\int_{0}^{t} \lambda(\mathcal{L} u(t)+\mathcal{N} u(t)-g(t)) d t
$$

a sufficient condition for convergence of the variational iteration method is strict contraction of $A$. Furthermore, the sequence (9) converges to the fixed point of $A$ which is also the solution of problem (4).

\subsection{VIM for $n$-th order FDE}

Consider the following $n$-th order fuzzy differential equation

$$
\begin{aligned}
& y^{(n)}+f\left(t, y(t), y^{\prime}(t), y^{\prime \prime}(t), \ldots, y^{(n)}(t)\right)=0 \\
& t \in[0,1] \\
& \widetilde{y}^{(i)}(0)=\left(g_{i}(r), k_{i}(r)\right), \quad i=0,1,2, \ldots, n-1 .
\end{aligned}
$$


The correction functionals for Eqs. (10) read:

$$
\begin{aligned}
& \underline{y}_{n+1}(t, r)=\underline{y}_{n}(t, r) \\
& +\int_{0}^{t} \underline{\lambda}\left\{\frac{d^{n}}{d s^{n}} \underline{y}_{n}+f\left(t, \underline{\tilde{y}}_{n}(s), \underline{\tilde{y}}_{n}^{\prime}(s), \ldots, \underline{\tilde{y}}_{n}^{(n)}(s)\right)\right\} d s, \\
& \bar{y}_{n+1}(t, r)=\bar{y}_{n}(t, r) \\
& +\int_{0}^{t} \bar{\lambda}\left\{\frac{d^{n}}{d s^{n}} \bar{y}_{n}+f\left(t, \tilde{\bar{y}}_{n}(s), \tilde{\bar{y}}_{n}^{\prime}(s), \ldots, \tilde{\bar{y}}_{n}^{(n)}(s)\right)\right\} d s \\
& n \geq 0 .
\end{aligned}
$$

In view of (7) we obtain the Lagrange multipliers in the form

$$
\underline{\lambda}(s, t)=\bar{\lambda}(s, t)=(-1)^{n} \frac{(s-t)^{(n-1)}}{(n-1) !} .
$$

It must be pointed out that if $f$ be a linear operator according to the Euler-Lagrange differential equations we can obtain the exact value of the Lagrange multipliers [37]. As a result, we obtain the following iteration formula

$$
\begin{aligned}
& \underline{y}_{n+1}(t, r)=\underline{y}_{n}(t, r)+\int_{0}^{t}(-1)^{n} \frac{(s-t)^{(n-1)}}{(n-1) !} \\
& \times\left\{\frac{d^{n}}{d s^{n}} \underline{y}_{n}+f\left(t, \underline{y}_{n}(s), \underline{y}_{n}^{\prime}(s), \ldots, \underline{y}_{n}^{(n)}(s)\right)\right\} d s, \\
& \bar{y}_{n+1}(t, r)=\bar{y}_{n}(t, r)+\int_{0}^{t}(-1)^{n} \frac{(s-t)^{(n-1)}}{(n-1) !} \\
& \times\left\{\frac{d^{n}}{d s^{n}} \bar{y}_{n}+f\left(t, \bar{y}_{n}(s), \bar{y}_{n}^{\prime}(s), \ldots, \bar{y}_{n}^{(n)}(s)\right)\right\} d s .
\end{aligned}
$$

According to Banach's fixed point theorem, it is easy to obtain the convergence condition for the sequences obtained from (13).

\section{Theorem 2.}

Define a nonlinear mapping

$$
\begin{aligned}
& T \underline{y}(t)]=\underline{y}(t, r) \\
& +\int_{0}^{t}(-1)^{n} \frac{(s-t)^{(n-1)}}{(n-1) !} \\
& \times\left\{\frac{d^{n}}{d s^{n}} \underline{y}+f\left(t, \underline{y}(s), \underline{y^{\prime}}(s), \ldots, \underline{y}^{(n)}(s)\right)\right\} d s, \\
& S[\bar{y}(t)]=\bar{y}(t, r) \\
& +\int_{0}^{t}(-1)^{n} \frac{(s-t)^{(n-1)}}{(n-1) !} \\
& \times\left\{\frac{d^{n}}{d s^{n}} \bar{y}+f\left(t, \bar{y}(s), \bar{y}^{\prime}(s), \ldots, \bar{y}^{(n)}(s)\right)\right\} d s .
\end{aligned}
$$

A sufficient condition for convergence of the iterative sequences $\underline{y}_{n}(t)$ and $\bar{y}_{n}(t)$ obtained from (13) is strict contraction of the nonlinear mappings $T$ and $S$. Furthermore, the sequence (13) converges to the fixed point of $T$ and $S$ which is also the solution of Eq. (10).

Therefore, according to (13), by choosing an initial approximation $\tilde{y}(0)=\left(g_{0}(r), k_{0}(r)\right)$, the successive approximations of the solution to (10) can be obtained.

For a linear fuzzy differential equation, the exact solution can be obtained due to the fact that the Lagrange multiplier can be exactly identified [29, 37].

\section{Numerical results}

To give a clear overview of the method and show the ability of the method, we present the following few examples.

\section{Example 1.}

[33]. Consider the following second-order linear fuzzy differential equation

$$
\left\{\begin{array}{l}
y^{\prime \prime}+y=-t, \quad t \in[0,1] \\
\widetilde{y}(0)=(0.1 r-0.1,0.1-0.1 r) \\
\widetilde{y}^{\prime}(0)=(0.088+0.1 r, 0.288-0.1 r)
\end{array}\right.
$$

with the exact fuzzy solution:

$$
\begin{aligned}
& \underline{Y}(t, r)=(0.1 r-0.1) \cos t+(1.088+0.1 r) \sin t-t, \\
& \bar{Y}(t, r)=(0.1-0.1 r) \cos t+(1.288+0.1 r) \sin t-t,
\end{aligned}
$$

To apply the VIM, first we rewrite Eq. (14) in the form

$$
L \underline{y}+N \underline{y}=0, \quad L \bar{y}+N \bar{y}=0
$$

where the notations $L \underline{y}=\frac{d^{2} \underline{y}}{d t^{2}}+\underline{y}, L \bar{y}=\frac{d^{2} \bar{y}}{d t^{2}}+\bar{y}, N \underline{y}=t$ and $N \bar{y}=t$ symbolize the linear and nonlinear terms, respectively. The correction functionals for Eqs. (16) read:

$$
\begin{gathered}
\underline{y}_{n+1}(t, r)=\underline{y}_{n}(t, r)+\int_{0}^{t} \underline{\lambda}\left\{\frac{d^{2}}{d s^{2}} \underline{y}_{n}+\underline{y}_{n}+N\left(\underline{\tilde{y}}_{n}\right)\right\} d s, \\
\bar{y}_{n+1}(t, r)=\bar{y}_{n}(t, r)+\int_{0}^{t} \bar{\lambda}\left\{\frac{d^{2}}{d s^{2}} \bar{y}_{n}+\bar{y}_{n}+N\left(\tilde{\bar{y}}_{n}\right)\right\} d s \\
n \geq 0 .
\end{gathered}
$$


Taking the variation with respect to the independent variables $\underline{y}_{n}$ and $\bar{y}$, and noticing that $\delta N\left(\underline{\widetilde{y}}_{n}(0)\right)=0, \delta N\left(\widetilde{\bar{y}}_{n}(0)\right)=0$ :

$$
\begin{aligned}
& \delta \underline{y}_{n+1}(t, r)=\delta \underline{y}_{n}(t, r) \\
& +\delta \int_{0}^{t} \underline{\lambda}\left\{\frac{d^{2}}{d s^{2}} \underline{y}_{n}+\underline{y}_{n}+N\left(\underline{\tilde{y}}_{n}\right)\right\} d s \\
& =\delta \underline{y}_{n}(t, r)-\left.\underline{\lambda}^{\prime} \delta \underline{y}_{n}\right|_{s=t}+\left.\underline{\lambda}^{\delta} \underline{y}_{n}^{\prime}\right|_{s=t} \\
& +\int_{0}^{t}\left\{\frac{\partial^{2} \underline{\lambda}}{\partial s^{2}}+\underline{\lambda}\right\} \delta \underline{y}_{n}(s) d s=0,
\end{aligned}
$$

$$
\begin{aligned}
& \delta \bar{y}_{n+1}(t, r)=\delta \bar{y}_{n}(t, r) \\
& +\delta \int_{0}^{t} \bar{\lambda}\left\{\frac{d^{2}}{d s^{2}} \bar{y}_{n}+\bar{y}_{n}+N\left(\tilde{\bar{y}}_{n}\right)\right\} d s \\
& =\delta \bar{y}_{n}(t, r)-\left.\bar{\lambda}^{\prime} \delta \bar{y}_{n}\right|_{s=t}+\left.\bar{\lambda} \delta \bar{y}_{n}^{\prime}\right|_{s=t} \\
& +\int_{0}^{t}\left\{\frac{\partial^{2} \bar{\lambda}}{\partial s^{2}}+\bar{\lambda}\right\} \delta \bar{y}_{n}(s) d s=0 .
\end{aligned}
$$

Thus, we obtain the Euler-Lagrange equations

$$
\begin{gathered}
\frac{\partial^{2} \underline{\lambda}(s, t)}{\partial s^{2}}+\underline{\lambda}(s, t)=0, \\
\frac{\partial^{2} \bar{\lambda}(s, t)}{\partial s^{2}}+\bar{\lambda}(s, t)=0
\end{gathered}
$$

and the natural boundary conditions:

$$
\begin{aligned}
1-\left.\underline{\lambda}^{\prime}(s, t)\right|_{s=t}=0, & 1-\left.\bar{\lambda}^{\prime}(s, t)\right|_{s=t} & =0, \\
\left.\underline{\lambda}(s, t)\right|_{s=t}=0, & \left.\bar{\lambda}(s, t)\right|_{s=t} & =0 .
\end{aligned}
$$

We, therefore, identify the Lagrange multiplier in the form

$$
\underline{\lambda}(s, t)=\sin (s-t), \quad \bar{\lambda}(s, t)=\sin (s-t) .
$$

Substituting the identified Lagrange multiplier into (17) results in the following iteration formulation:

$\underline{y}_{n+1}(t, r)=\underline{y}_{n}(t, r)+\int_{0}^{t} \sin (s-t)\left\{\frac{d^{2}}{d s^{2}} \underline{y}_{n}+\underline{y}_{n}+s\right\} d s$,

$\bar{y}_{n+1}(t, r)=\bar{y}_{n}(t, r)+\int_{0}^{t} \sin (s-t)\left\{\frac{d^{2}}{d s^{2}} \bar{y}_{n}+\bar{y}_{n}+s\right\} d s$

$n \geq 0$.
If we begin with $\underline{y}_{0}(t, r)=(0.1 r-0.1)+t(0.088+0.1 r)$ and $\bar{y}_{0}(t, r)=(0.1-0.1 r)+t(0.288-0.1 r)$, then

$$
\begin{aligned}
& \underline{y}_{2}=(0.1 r-0.1) \cos t+(1.088+0.1 r) \sin t-t, \\
& \bar{y}_{2}=(0.1-0.1 r) \cos t+(1.288+0.1 r) \sin t-t,
\end{aligned}
$$

which is the exact explicit solution.

\section{Example 2.}

[33]. Consider the following second-order fuzzy linear differential equation

$$
\left\{\begin{array}{l}
y^{\prime \prime}-4 y^{\prime}+4 y=0, \quad t \in[0,1] \\
\widetilde{y}(0)=(2+r, 4-r) \\
\widetilde{y}^{\prime}(0)=(5+r, 7-r)
\end{array}\right.
$$

The exact solution is as follows:

$$
\begin{aligned}
& \underline{Y}(t, r)=(2+r) e^{2 t}+(1-r) t e^{2 t}, \\
& \bar{Y}(t, r)=(4-r) e^{2 t}+(r-1) t e^{2 t} .
\end{aligned}
$$

To apply the VIM, first we rewrite Eq. (22) in the form

$$
L \underline{y}+N \underline{y}=0, \quad L \bar{y}+N \bar{y}=0
$$

where the notations $L \underline{y}=\frac{d^{2} y}{d t^{2}}, L \bar{y}=\frac{d^{2} \bar{y}}{d t^{2}}, N \underline{y}=-4 \frac{d \underline{y}}{d t}+4 \underline{y}$ and $N \bar{y}=-4 \frac{d \bar{y}}{d t}+4 \bar{y}$ symbolize the linear and nonlinear terms, respectively. The correction functionals for Eqs. (24) read

$$
\begin{aligned}
& \underline{y}_{n+1}(t, r)=\underline{y}_{n}(t, r)+\int_{0}^{t} \underline{\lambda}\left\{\frac{d^{2}}{d s^{2}} \underline{y}_{n}+N\left(\underline{\tilde{y}}_{n}\right)\right\} d s, \\
& \bar{y}_{n+1}(t, r)=\bar{y}_{n}(t, r)+\int_{0}^{t} \bar{\lambda}\left\{\frac{d^{2}}{d s^{2}} \bar{y}_{n}+N\left(\tilde{\bar{y}}_{n}\right)\right\} d s \quad n \geq 0
\end{aligned}
$$

Taking the variation with respect to the independent variables $\underline{y}_{n}$ and $\bar{y}$, and noticing that $\delta N\left(\underline{\tilde{y}}_{n}(0)\right)=$ $0, \delta N\left(\widetilde{\bar{y}}_{n}(0)\right)=0$ :

$$
\begin{aligned}
& \delta \underline{y}_{n+1}(t, r)=\delta \underline{y}_{n}(t, r) \\
& +\delta \int_{0}^{t} \underline{\lambda}\left\{\frac{d^{2}}{d s^{2}} \underline{y}_{n}+N\left(\underline{\tilde{y}}_{n}\right)\right\} d s \\
& =\delta \underline{y}_{n}(t, r)-\left.\underline{\lambda}^{\prime} \delta \underline{y}_{n}\right|_{s=t}+\left.\underline{\lambda}^{\prime} \underline{y}_{n}^{\prime}\right|_{s=t} \\
& +\int_{0}^{t}\left\{\frac{\partial^{2} \underline{\lambda}}{\partial s^{2}}\right\} \delta \underline{y}_{n}(s) d s=0,
\end{aligned}
$$




$$
\begin{aligned}
& \delta \bar{y}_{n+1}(t, r)=\delta \bar{y}_{n}(t, r)+\delta \int_{0}^{t} \bar{\lambda}\left\{\frac{d^{2}}{d s^{2}} \bar{y}_{n}+N\left(\tilde{\bar{y}}_{n}\right)\right\} d s \\
& =\delta \bar{y}_{n}(t, r)-\left.\bar{\lambda}^{\prime} \delta \bar{y}_{n}\right|_{s=t}+\left.\bar{\lambda} \delta \bar{y}_{n}^{\prime}\right|_{s=t} \\
& +\int_{0}^{t}\left\{\frac{\partial^{2} \bar{\lambda}}{\partial s^{2}}\right\} \delta \bar{y}_{n}(s) d s=0 .
\end{aligned}
$$

Thus, we obtain the Euler-Lagrange equations

$$
\begin{aligned}
& \frac{\partial^{2} \underline{\lambda}(s, t)}{\partial s^{2}}=0, \\
& \frac{\partial^{2} \bar{\lambda}(s, t)}{\partial s^{2}}=0
\end{aligned}
$$

and the natural boundary conditions:

$$
\begin{array}{rlrl}
1-\left.\underline{\lambda}^{\prime}(s, t)\right|_{s=t} & =0, & 1-\left.\bar{\lambda}^{\prime}(s, t)\right|_{s=t} & =0, \\
\left.\underline{\lambda}(s, t)\right|_{s=t}=0, & \left.\bar{\lambda}(s, t)\right|_{s=t} & =0 .
\end{array}
$$

We, therefore, identify the Lagrange multiplier in the form

$$
\underline{\lambda}(s, t)=(s-t), \quad \bar{\lambda}(s, t)=(s-t) .
$$

Substituting the identified Lagrange multiplier into (25) results in the following iteration formulation:

$$
\begin{aligned}
& \underline{y}_{n+1}(t, r)=\underline{y}_{n}(t, r) \\
& +\int_{0}^{t}(s-t)\left\{\frac{d^{2}}{d s^{2}} \underline{y}_{n}-4 \frac{d}{d s} \underline{y}_{n}+4 \underline{y}_{n}\right\} d s \\
& \bar{y}_{n+1}(t, r)=\bar{y}_{n}(t, r) \\
& +\int_{0}^{t}(s-t)\left\{\frac{d^{2}}{d s^{2}} \bar{y}_{n}-4 \frac{d}{d s} \bar{y}_{n}+\bar{y}_{n}\right\} d s \quad n \geq 0 .
\end{aligned}
$$

Choosing $\underline{y}_{0}(t, r)=(2+r)+t(5+r)$ and $\bar{y}_{0}(t, r)=(4-$ $r)+t(7-r)$, after 3 iterations we obtained approximate solutions:

$$
\begin{aligned}
& \underline{y} \approx \underline{y}_{3}=(2+r)+(5+r) t+6 t^{2}+\left(\frac{14}{13}-\frac{2}{13} r\right) t^{3} \\
& -\left(\frac{16}{3}+\frac{2}{3} r\right) t^{4}-\left(\frac{2}{3}+\frac{2}{15} r\right) t^{5} \\
& \bar{y} \approx \bar{y}_{3}=(4-r)+(7-r) t+6 t^{2}+\left(\frac{10}{3}+\frac{2}{3} r\right) t^{3}- \\
& \left(\frac{20}{3}-\frac{2}{3} r\right) t^{4}+\left(\frac{14}{15}-\frac{2}{15} r\right) t^{5}
\end{aligned}
$$

We compare our results with the numerical solutions from [33] in Tables 1 and 2.
Table 1. For $\underline{y}(t=0.01)$

\begin{tabular}{ccc}
\hline$r$ & error(VIM) & error [33] \\
\hline \hline 0.0 & $0.800538 \mathrm{e}-9$ & $0.461836 \mathrm{e}-3$ \\
0.1 & $0.800482 \mathrm{e}-9$ & $0.476055 \mathrm{e}-3$ \\
0.2 & $0.800431 \mathrm{e}-9$ & $0.490273 \mathrm{e}-3$ \\
0.3 & $0.800377 \mathrm{e}-9$ & $0.504491 \mathrm{e}-3$ \\
0.4 & $0.800324 \mathrm{e}-9$ & $0.518710 \mathrm{e}-3$ \\
0.5 & $0.800270 \mathrm{e}-9$ & $0.532928 \mathrm{e}-3$ \\
0.6 & $0.800217 \mathrm{e}-9$ & $0.547146 \mathrm{e}-3$ \\
0.7 & $0.800163 \mathrm{e}-9$ & $0.561365 \mathrm{e}-3$ \\
0.8 & $0.800110 \mathrm{e}-9$ & $0.575584 \mathrm{e}-3$ \\
0.9 & $0.800056 \mathrm{e}-9$ & $0.589801 \mathrm{e}-3$ \\
1.0 & $0.800003 \mathrm{e}-9$ & $0.604020 \mathrm{e}-4$ \\
\hline
\end{tabular}

Table 2. For $\bar{y}(t=0.01)$

\begin{tabular}{ccc}
\hline$r$ & error(VIM) & error [33] \\
\hline \hline 0.0 & $0.799467 \mathrm{e}-9$ & $0.746204 \mathrm{e}-3$ \\
0.1 & $0.799521 \mathrm{e}-9$ & $0.731985 \mathrm{e}-3$ \\
0.2 & $0.799574 \mathrm{e}-9$ & $0.717767 \mathrm{e}-3$ \\
0.3 & $0.799628 \mathrm{e}-9$ & $0.703549 \mathrm{e}-3$ \\
0.4 & $0.799682 \mathrm{e}-9$ & $0.689330 \mathrm{e}-3$ \\
0.5 & $0.799735 \mathrm{e}-9$ & $0.675112 \mathrm{e}-3$ \\
0.6 & $0.799789 \mathrm{e}-9$ & $0.660894 \mathrm{e}-3$ \\
0.7 & $0.799842 \mathrm{e}-9$ & $0.646675 \mathrm{e}-3$ \\
0.8 & $0.799896 \mathrm{e}-9$ & $0.632456 \mathrm{e}-3$ \\
0.9 & $0.799949 \mathrm{e}-9$ & $0.618239 \mathrm{e}-3$ \\
1.0 & $0.800002 \mathrm{e}-9$ & $0.604020 \mathrm{e}-3$ \\
\hline
\end{tabular}

\section{Example 3.}

Electrical circuit [33], If $Q$ is the charge on the capacitor at time $t>0$, then

$$
Q^{\prime \prime}+2 Q^{\prime}+4 Q=50 \cos t
$$

for $\widetilde{Q}(0)=(4+r, 6-r), \widetilde{Q}^{\prime}(0)=(r, 2-r)$.

To apply the VIM, first we rewrite Eq. (30) in the form

$$
L \underline{y}+N \underline{y}=0, \quad L \bar{y}+N \bar{y}=0
$$

where the notations $L \underline{Q}=\frac{d^{2} Q}{d t^{2}}+4 \underline{Q}, L \bar{Q}=\frac{d^{2} \bar{Q}}{d t^{2}}+4 \bar{Q}$, $N \underline{Q}=2 \frac{d \underline{Q}}{d t}-50 \cos t$ and $N \bar{Q}=2 \frac{d \bar{Q}}{d t}-50 \cos t$ symbolize 
the linear and nonlinear terms, respectively. The correction functionals for Eq. (31) reads

$$
\begin{aligned}
& \underline{Q}_{n+1}(t, r)=\underline{Q}_{n}(t, r) \\
& +\int_{0}^{t} \underline{\lambda}\left\{\frac{d^{2}}{d s^{2}} \underline{Q}_{n}+4 \underline{Q}_{n}+N\left(\underline{Q}_{n}\right)\right\} d s \\
& \bar{Q}_{n+1}(t, r)=\bar{Q}_{n}(t, r) \\
& +\int_{0}^{t} \bar{\lambda}\left\{\frac{d^{2}}{d s^{2}} \bar{Q}_{n}+4 \bar{Q}_{n}+N\left(\tilde{\bar{Q}}_{n}\right)\right\} d s \quad n \geq 0 .
\end{aligned}
$$

Taking the variation with respect to the independent variable $\underline{Q}_{n}, \bar{Q}$, and noticing that $\delta N\left(\underline{\widetilde{Q}}_{n}(0)\right)=0, \delta N\left(\widetilde{\bar{Q}}_{n}(0)\right)=$ 0 :

$$
\begin{aligned}
& \delta \underline{Q}_{n+1}(t, r)=\delta \underline{Q}_{n}(t, r) \\
& +\delta \int_{0}^{t} \underline{\lambda}\left\{\frac{d}{d s} \underline{Q}_{n}+4 \underline{Q}_{n}+N\left(\underline{\tilde{Q}}_{n}\right)\right\} d s \\
& =\delta \underline{Q}_{n}(t, r)-\left.\bar{\lambda}^{\prime} \delta \bar{Q}_{n}\right|_{s=t}+\left.\bar{\lambda} \delta \bar{Q}_{n}^{\prime}\right|_{s=t} \\
& +\int_{0}^{t}\left\{\frac{\partial \underline{\lambda}}{\partial s}+4 \underline{\lambda}\right\} \delta \underline{Q}_{n}(s, r) d s=0, \\
& \delta \bar{Q}_{n+1}(t, r)=\delta \bar{Q}_{n}(t, r) \\
& +\delta \int_{0}^{t} \bar{\lambda}\left\{\frac{d^{2}}{d s^{2}} \bar{Q}_{n}+4 \bar{Q}_{n}+N\left(\tilde{\bar{Q}}_{n}\right)\right\} d s \\
& =\delta \bar{Q}_{n}(t, r)-\left.\bar{\lambda}^{\prime} \delta \bar{Q}_{n}\right|_{s=t}+\left.\bar{\lambda} \delta \bar{Q}_{n}^{\prime}\right|_{s=t} \\
& +\int_{0}^{t}\left\{\frac{\partial \bar{\lambda}}{\partial s}+4 \bar{\lambda}\right\} \delta \bar{Q}_{n}(s, r) d s=0 .
\end{aligned}
$$

Thus, we obtain the Euler-Lagrange equations

$$
\begin{aligned}
& \frac{\partial \underline{\lambda}(s, t)}{\partial s}+4 \underline{\lambda}(s, t)=0 \\
& \frac{\partial \bar{\lambda}(s, t)}{\partial s}+4 \bar{\lambda}(s, t)=0
\end{aligned}
$$

and the natural boundary conditions:

$$
\begin{aligned}
1-\left.\underline{\lambda}^{\prime}(s, t)\right|_{s=t}=0, & 1-\left.\bar{\lambda}^{\prime}(s, t)\right|_{s=t} & =0, \\
\left.\underline{\lambda}(s, t)\right|_{s=t}=0, & \left.\bar{\lambda}(s, t)\right|_{s=t} & =0 .
\end{aligned}
$$

We, therefore, identify the Lagrange multiplier in the form

$$
\underline{\lambda}(s, t)=\frac{1}{2} \sin (2(s-t)), \quad \bar{\lambda}(s, t)=\frac{1}{2} \sin (2(s-t)) .
$$

Table 3. For $\underline{Q}(t=0.001)$

\begin{tabular}{ccc}
\hline$r$ & error(VIM) & error [33] \\
\hline \hline 0.0 & $0.199800 \mathrm{e}-4$ & $0.470675 \mathrm{e}-3$ \\
0.1 & $0.179820 \mathrm{e}-4$ & $0.339847 \mathrm{e}-3$ \\
0.2 & $0.159840 \mathrm{e}-4$ & $0.209017 \mathrm{e}-3$ \\
0.3 & $0.139860 \mathrm{e}-4$ & $0.781880 \mathrm{e}-4$ \\
0.4 & $0.119880 \mathrm{e}-4$ & $-0.526410 \mathrm{e}-4$ \\
0.5 & $0.999000 \mathrm{e}-5$ & $-0.183471 \mathrm{e}-3$ \\
0.6 & $0.799200 \mathrm{e}-5$ & $-0.314300 \mathrm{e}-3$ \\
0.7 & $0.599401 \mathrm{e}-5$ & $-0.445130 \mathrm{e}-3$ \\
0.8 & $0.399601 \mathrm{e}-5$ & $-0.575959 \mathrm{e}-3$ \\
0.9 & $0.199801 \mathrm{e}-5$ & $-0.706788 \mathrm{e}-3$ \\
1.0 & $0.100641 \mathrm{e}-10$ & $-0.837618 \mathrm{e}-3$ \\
\hline
\end{tabular}

Substituting the identified Lagrange multiplier into (32) results in the following iteration formulation:

$$
\begin{aligned}
& \underline{Q}_{n+1}(t, r)=\underline{Q}_{n}(t, r)+\int_{0}^{t} \frac{1}{2} \sin (2(s-t)) \\
& \times\left\{\frac{d^{2}}{d s^{2}} \underline{Q}_{n}+2 \frac{d}{d s} \underline{Q}_{n}+4 \underline{Q}_{n}-50 \cos t\right\} d s, \\
& \bar{Q}_{n+1}(t, r)=\bar{Q}_{n}(t, r)+\int_{0}^{t} \frac{1}{2} \sin (2(s-t)) \\
& \times\left\{\frac{d^{2}}{d s^{2}} \bar{Q}_{n}+2 \frac{d}{d s} \bar{Q}_{n}+4 \bar{Q}_{n}-50 \cos t\right\} d s, \\
& n \geq 0 .
\end{aligned}
$$

With the choices $\underline{Q}_{0}(t, r)=4+r+t r$ and $\bar{Q}_{0}(t, r)=$ $6-r+t(2-r)$, in 3 iteration we obtained the approximate solutions:

$$
\begin{aligned}
\underline{Q} & =\frac{50}{3} \cos t+\left(-\frac{38}{3}+r-\frac{3}{2} t r+\frac{38}{3} t\right) \cos 2 t \\
& +\frac{100}{9} \sin t+\left(-\frac{107}{9}+\frac{5}{4} r-\frac{1}{2} t r\right) \sin 2 t \\
\bar{Q} & =\frac{50}{3} \cos t+\left(-\frac{32}{3}-r+\frac{3}{2} t r+\frac{29}{3} t\right) \cos 2 t \\
& +\frac{100}{9} \sin t+\left(-\frac{169}{18}-\frac{5}{4} r+\frac{1}{2} t r-t\right) \sin 2 t
\end{aligned}
$$

We compare our results with the numerical solutions from [33] in Tables 3 and 4.

\section{Example 4.}

[39]. Consider the following fourth-order fuzzy linear dif- 
ferential equation

$$
\begin{aligned}
& y^{(4)}(t)-y=0, \quad t \in[0,1] \\
& \widetilde{y}(0)=(r-1,1-r), \widetilde{y}^{\prime}(0)=(r-1,1-r), \\
& \widetilde{y}^{\prime \prime}(0)=(r-1,1-r), \widetilde{y}^{\prime \prime \prime}(0)=(r-1,1-r) .
\end{aligned}
$$

with the exact fuzzy solution:

$$
\begin{aligned}
& \underline{Y}(t, r)=(r-1) e^{t}, \\
& \bar{Y}(t, r)=(1-r) e^{t},
\end{aligned}
$$

To apply the VIM, first we rewrite Eq. (37) in the form

$$
L \underline{y}+N \underline{y}=0, \quad L \bar{y}+N \bar{y}=0
$$

where the notations $L \underline{y}=\frac{d^{4} \underline{y}}{d t^{4}}-\underline{y}, L \bar{y}=\frac{d^{4} \bar{y}}{d t^{4}}-\bar{y}, N \underline{y}=0$ and $N \bar{y}=0$ symbolize the linear and nonlinear terms, respectively. The correction functionals for Eq. (39) reads

$$
\begin{aligned}
& \underline{y}_{n+1}(t, r)=\underline{y}_{n}(t, r)+\int_{0}^{t} \underline{\lambda}\left\{\frac{d^{4}}{d s^{4}} \underline{y}_{n}-\underline{y}\right\} d s, \\
& \bar{y}_{n+1}(t, r)=\bar{y}_{n}(t, r)+\int_{0}^{t} \bar{\lambda}\left\{\frac{d^{4}}{d s^{4}} \bar{y}_{n}-\bar{y}\right\} d s, \\
& n \geq 0 .
\end{aligned}
$$

According to algorithm given by Jafari and Alipoor [37] for finding $\lambda$ in the VIM we have

$$
\left\{\begin{array}{l}
-\underline{\lambda}(s)+\underline{\lambda}^{(4)}(s)=0 \\
1-\left.\underline{\lambda}^{\prime \prime \prime}(s)\right|_{s=t}=0 \\
\left.\underline{\lambda}^{\prime \prime}(s)\right|_{s=t}=0, \\
\left.\underline{\lambda}^{\prime}(s)\right|_{s=t}=0, \\
\left.\underline{\lambda}\right|_{s=t}=0,
\end{array}, \quad\left\{\begin{array}{l}
-\bar{\lambda}(s)+\bar{\lambda}^{(4)}(s)=0 \\
1-\left.\bar{\lambda}^{\prime \prime \prime}(s)\right|_{s=t}=0 \\
\left.\bar{\lambda}^{\prime \prime}(s)\right|_{s=t}=0, \\
\left.\bar{\lambda}^{\prime}(s)\right|_{s=t}=0, \\
\left.\bar{\lambda}(s)\right|_{s=t}=0,
\end{array}\right.\right.
$$

By solving equations (41), the Lagrange multipliers can be identified as

$\bar{\lambda}=\underline{\lambda}=\frac{1}{2}[\sinh (s-x)-\sin (s-t)]$, and the following variational iteration formula can be obtained:

$$
\begin{aligned}
& \underline{y}_{n+1}(t, r)=\underline{y}_{n}(t, r) \\
& +\int_{0}^{t}[\sinh (s-x)-\sin (s-t)]\left\{\frac{d^{4}}{d s^{4}} \underline{y}_{n}-\underline{y}\right\} d s, \\
& \bar{y}_{n+1}(t, r)=\bar{y}_{n}(t, r) \\
& +\int_{0}^{t}[\sinh (s-x)-\sin (s-t)]\left\{\frac{d^{4}}{d s^{4}} \bar{y}_{n}-\bar{y}\right\} d s, \\
& n \geq 0 .
\end{aligned}
$$

Table 4. For $\bar{Q}(t=0.001)$

\begin{tabular}{ccc}
\hline$r$ & error(VIM) & error [33] \\
\hline \hline 0.0 & $0.199800 \mathrm{e}-4$ & $-0.2145913 \mathrm{e}-2$ \\
0.1 & $0.179820 \mathrm{e}-4$ & $-0.2015082 \mathrm{e}-2$ \\
0.2 & $0.159840 \mathrm{e}-4$ & $-0.1884252 \mathrm{e}-2$ \\
0.3 & $0.139860 \mathrm{e}-4$ & $-0.1753422 \mathrm{e}-2$ \\
0.4 & $0.119880 \mathrm{e}-4$ & $-0.1622592 \mathrm{e}-2$ \\
0.5 & $0.998999 \mathrm{e}-5$ & $-0.1491763 \mathrm{e}-2$ \\
0.6 & $0.799199 \mathrm{e}-5$ & $-0.1360935 \mathrm{e}-2$ \\
0.7 & $0.599399 \mathrm{e}-5$ & $-0.1230105 \mathrm{e}-2$ \\
0.8 & $0.399599 \mathrm{e}-5$ & $-0.1099277 \mathrm{e}-2$ \\
0.9 & $0.199799 \mathrm{e}-5$ & $-0.9684460 \mathrm{e}-3$ \\
1.0 & $0.100641 \mathrm{e}-10$ & $-0.8376160 \mathrm{e}-3$ \\
\hline
\end{tabular}

With the choices $\underline{y}_{0}(t, r)=(r-1)\left(1+t+\frac{t^{2}}{2 !}+\frac{t^{3}}{3 !}\right)$ and $\bar{y}_{0}(t, r)=(1-r)\left(1+t+\frac{t^{2}}{2 !}+\frac{t^{3}}{3 !}\right)$ we obtained

$$
\begin{array}{cc}
\underline{y}_{1}=(r-1) e^{t}, & \bar{y}_{1}=(1-r) e^{t}, \\
\underline{y}_{2}=(r-1) e^{t}, & \bar{y}_{2}=(1-r) e^{t}, \\
\vdots & \vdots \\
\underline{y}_{n}=(r-1) e^{t}, & \bar{y}_{n}=(1-r) e^{t} .
\end{array}
$$

Therefore $\underline{Y}(t, r)=(r-1) e^{t}, \bar{Y}(t, r)=(1-r) e^{t}$, which is the exact solution.

Remark. We find the exact solution after one iteration but Abbasbandy et al. [39] find approximate solutions after 10 iterations. The algorithm in [39] yields a system of 4 equations corresponding to Eq. (37), whereas according to the formulation in the present paper, Eq. (37) is equivalent to the one FDE only.

\section{Conclusion}

In this paper, a variational iteration method (VIM) has been successfully applied to finding solutions of $n$-th order linear fuzzy differential equations. The solution obtained by the variational iteration method is an infinite power series, which, with appropriate initial condition, can be expressed in a closed form, i.e. the exact solution. The results presented in this contribution show that the variational iteration method is a powerful mathematical tool 
to solving $n$-th order fuzzy differential equations. For linear fuzzy differential equation, its exact solution can be obtained by the VIM due to the fact that the Lagrange multiplier can be exactly identified. We have compared our approach with the algorithm given in [39] by Abbasbandy et al.. They converted the $n$-th order linear fuzzy differential equation to a system of linear fuzzy differential equations which contains $n$ fuzzy differential equations of order one, whereas we directly applied the VIM for this type of equation. Convergence of the VIM has been discussed for $n$-th order fuzzy differential equations. It is also a promising method to solve other linear equations.

\section{Acknowledgment}

The authors express their gratitude to the referees for their valuable suggestions and corrections for improvement of this paper. This research is partially supported by the Research Center on Algebraic Hyperstructures and Fuzzy Mathematics, University of Mazandaran, Babolsar, Iran and the National Elite Foundation of Iran.

\section{References}

[1] A. Kandel, In: P. P. Wang, S. K. Chang (Eds.), Fuzzy sets theory and application to policy analysis and information systems (Plenum Press, New York, 1980) 93

[2] A. Kandel, W. J. Byatt, Proceedings of the international conference on cybernetics and society, Nov. 1978, Tokyo, 1213

[3] M. A. Abdou, A. A. Soliman, J. Comput. Appl. Math. 181, 245 (2005)

[4] M. A. Abdou, A. A. Soliman, Physica D 211, 1 (2005)

[5] S. Momani, S. Abuasad, Chaos, Soliton. Fract. 27, 119 (2006)

[6] H. T. Nguyen, J. Math. Anal. Appl. 64, 369 (1978)

[7] J. H. He, J. Comput. Appl. Math. 207, 3 (2007)

[8] J. H. He, Chaos Soliton. Fract. 19, 847 (2004)

[9] J. H. He, Appl. Math. Comput. 114, 115 (2000)

[10] J. H. He, Int. J. Mod. Phys. B 20, 1141 (2006)

[11] S. Q. Wang, J. H. He, Phys. Lett. A 367, 188 (2007)

[12] M. Inokuti et al., In: S. Nemat-Nasser (Ed.), Variational method in the mechanics of solids (Pergamon
Press, Oxford, 1978) 156

[13] A. M. Wazwaz, Comput. Math. Appl. 54, 926 (2007)

[14] A. M. Wazwaz, J. Comput. Appl. Math. 207, 18 (2007)

[15] B. Bede, I. J. Rudas, A. L. Bencsik, Inform. Sciences 177, 1648 (2007)

[16] D. Dubois, H. Prade, Fuzzy Set. Syst. 8, 225 (1982)

[17] J. Y. Park, Y. C. Kwan, J. V. Jeong, Fuzzy Set. Syst. 72, 373 (1995)

[18] J. J. Buckley, T. Feuring, Fuzzy Set. Syst. 121, 247 (2001)

[19] J. J. Buckley, Simulating continuous fuzzy systems (Springer, Heildelberg, 2006)

[20] J. J. Buckley, T. Feuring, Fuzzy Set. Syst. 105, 241 (1999)

[21] J. J. Buckley, T. Feuring, Fuzzy Set. Syst. 110, 43 (2000)

[22] L. J. Jowers, J. J. Buckley, K. D. Reilly, Inform. Sciences 177, 436 (2007)

[23] O. Kaleva, Fuzzy Set. Syst. 35, 389 (1990)

[24] O. Kaleva, Fuzzy Set. Syst. 56, 297 (1993)

[25] Z. Ding, M. Ma, A. Kandel, Inform. Sciences 99, 205 (1997)

[26] O. He, W. Yi, Fuzzy Set. Syst. 24, 321 (1989).

[27] O. Kaleva, Fuzzy Set. Syst. 24, 301 (1987)

[28] P. Kloeden, Fuzzy Set. Syst. 44, 161 (1991)

[29] R. Goetschel, W. Voxman, Fuzzy Set. Syst. 18, 31 (1986)

[30] S. Abbasbandy, T. Allahviranloo, Comput. Meth. Appl. Math. 2, 113 (2002)

[31] S. Seikkala, Fuzzy Set. Syst. 24, 319 (1987)

[32] S. L. Chang, L. A. Zadeh, IEEE T. Syst. Man Cy. 2, 330 (1972)

[33] T. Allahviranloo, E. Ahmady, N. Ahmady, Inform. Sciences 178, 1309 (2008)

[34] T. Allahviranloo, N. Ahmady, E. Ahmady, Inform. Sciences 177, 1633 (2007)

[35] W. Congxin, S. Shiji, Inform. Sciences 108, 123 (1998)

[36] W. Menda, J. Fuzzy Syst. Math. 2, 51 (1988) (in Chinese)

[37] H. Jafari, A. Alipoor, Numer. Meth. Part. D. E. 27, 996 (2011)

[38] A. M. Wazwaz, Int. J. Comput. Math. 87, 1131 (2010)

[39] S. Abbasbandy, T. Allahviranloo, P. Darabi, 
O. Sedaghatfar, Math. Comput. Appl. 16, 819 (2011) 